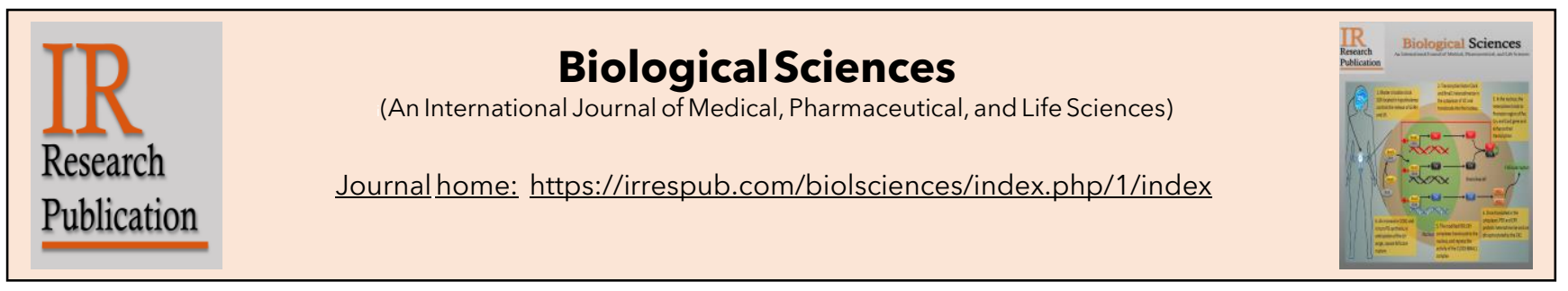

\title{
Chicken consumption predisposes to diabetes through epigenetic modification by miRNAs: an in-silico prediction
}

\author{
Ishfaq Ahmad Bhat ${ }^{*}$, Aasiya Bashir ${ }^{2}$, Lahanya Guha ${ }^{3}$, Ahmar Khan², Farheen Azad ${ }^{4}$ \\ ${ }^{1}$ Northern Railway Hospital, Associated Sri Mata Vaishno-Devi Narayana Superspeciality Hospital (SVDNSH), Katra, \\ J\&K, India, 182320 \\ 2Department of Pharmaceutical Sciences, Faculty of Applied Sciences and Technology, University of Kashmir, \\ Hazratbal, Srinagar-190006, J\&K, India \\ ${ }^{3}$ Department of Pharmacology and Toxicology, National Institute of Pharmaceutical Education and Research, S.A.S. \\ Nagar (Mohali), Punjab-160062, India \\ ${ }^{4}$ Department of Pharmacognosy and Phytochemistry, SPER, Jamia Hamdard University, New Delhi, 110062, India
}

\section{ARTICLEHISTORY}

Received: 02-04-2021

Revised: 04-04-2021

Accepted: 04-04-2021

Online: 0-04-2021

\section{KEYWORDS}

MicroRNA

Epigenetics

Diabetes

Chicken meat

In-silico

\section{ABSTRACT}

Diabetes is among the most worldwide occurring metabolic disorders. It has created a substantial socioeconomic burden because of no targeted therapy available to date. Epigenetic modifications are playing a significant role in its cause and cure. The food we eat can lead to epigenetic changes, which further can alter the expression of different genes. In the current research, we postulate that meat consumers might have a high risk of diabetes due to miRNA's ability to instigate epigenetic modifications of genes involved in diabetes. We performed bioinformatics analysis in which first we retrieved chicken miRNAs data from miRbase. Then we checked the interaction with target genes IGF2BP2, HNF1B, and TCF7L2 of obtained miRNAs utilizing miRanda and TargetScan databases. The interaction score obtained indicated good interaction between them. Based on the predicted miRNA-mRNA interactions score, we drew a miRNA network using miRNet software. The results obtained from this in-silico analysis suggest that chicken consumption may predispose diabetes through miRNAs associated with epigenetic modifications of the gene involved in diabetes. The outcome of this study can be further explored in cell-based, animal-based, and human-based studies.
*Address for correspondence

Northern Railway Hospital, Associated Sri Mata Vaishno-Devi Narayana Superspeciality Hospital (SVDNSH), Katra, J\&K, India, 182320

Email: ishfaqpsst13@gmail.com

DOI: http://dx.doi.org/10.55006/biolsciences.2021.1101 Published by IR Research Publication; Bhat A et al (C) 2021 by Biological Sciences is licensed under CC BY 4.0 (C)

\section{Introduction}

Diabetes is one of the most prevalent chronic diseases. The affected population is still growing, and it is estimated that $4-5$ million people will develop diabetes by 2030 (Guariguata et al., 2014; Cho et al., 2019). A combination of genetic, epigenetic, environmental, and lifestyle factors, such as diet, are responsible for the onset and development of T2DM (Ortega et al., 2017). Although we have not completely elucidated the 
pathophysiology of T2DM so far, it is the case that the disease has a major genetic component. So far, vital genes associated with GWAS of different populations include IGF2BP2, SLC30A8, TCF7L2, HNF1B, HHEX, KCNQ11, CDKN2A/B, HMGA2, and $\mathrm{NOTCH} 2-\mathrm{ADAM} 30$ are involved as a major factor in the pathology of T2D (Pradhan et al., 2016). Higher concordance rates are found among monozygotic (96\%) than dizygotic (DZ) twins in some not all twin studies, which has been compelling evidence of a significant genetic component in T2DM (Poulsen et al., 1999, 2009). Moreover, 40\% of first-degree relatives of T2DM patients may develop diabetes, whereas the incident rate is only $6 \%$ in the general population (Kobberling, 1982). MicroRNAs (miRNAs) an epigenetic regulator, are noncoding RNAs of $\sim 22$ nucleotides that regulate gene expression post-transcriptionally by binding to the 3' untranslated region of messenger RNA (mRNAs), resulting in inhibition of translation or mRNA degradation (Hashimoto and Tanaka, 2017). miRNA shows participation in the $\beta$ cell biology, insulin resistance, type | and type || diabetes mellitus, and its various complication in the whole anatomy and physiology of the human being (Tiwari et al., 2018).

Dietary nutrition plays an important role in the pathogenesis of T2DM, making the identification and analysis of nutrient-gene interactions a necessary step in the understanding of this chronic disease. Nutrigenomics, the area of nutrition that uses molecular tools to elucidate the influence of nutrients over the genome, proteome, and metabolome, provides a genetic understanding of how common dietary components lead to up and/or down-regulation of the gene (s) and changes in protein expression levels (Rana et al., 2016). Although several studies have confirmed a strong association between meat consumption and susceptibility to T2DM and other metabolic disorders, including obesity, atherosclerosis, and even cancer (Vang et al., 2008; Aune et al., 2009; Pan et al., 2011), it has not yet been described as a diabetes risk factor.

In this study, we hypothesized that miRNAs present in chicken meat might regulate genes associated with diabetes. To confirm this hypothesis, we performed bioinformatics analysis in which first we retrieved chicken miRNAs data from miRbase, and then we checked the target genes of obtained miRNAs utilizing miRanda and TargetScan databases. Based on the predicted miRNA-mRNA interactions score, we drew a miRNA network utilizing miRNet software. The results obtained from this in silicon analysis suggest that chicken consumption may predispose diabetes through miRNAs associated with epigenetic modifications of the gene involved in diabetes. Hence this study gives insight into the future finding of these associations in cell-based assays, animal, and human models.

\section{Materials and Methods}

We used three different web-based bioinformatic algorithms to predict the miRNAs that target three different genes involved in diabetes. The programs are:

miRbase (http://www.mirbase.org/)

miRanda (http://mirdb.org/)

TargetScan7.2

(http://www.targetscan.org/vert_72/)

miRNet (https://www.mirnet.ca/) 
The data of miRNAs present in Gallus gallus (Chicken) was retrieved from miRbase, and their regulation of three different genes, IGF2BP2, HNF1B, TCF7L2, was predicted based on interaction scores from two software miRanda \& Targetscan7.2. Further interaction diagram was obtained from software miRNet.

\section{Results and Discussion}

As per the OECD meat consumption data, $14.20285 \mathrm{Kg} /$ capita poultry, $12.31853 \mathrm{~kg} / \mathrm{capita}$ pig, 6.508807 kg/capita beef, 1.715142 kg/capita sheep are consumed in 2018 and estimated to rise in coming decades. Several studies have confirmed the strong association between meat consumption and susceptibility to T2DM and other metabolic disorders (Vang et al., 2008; Aune et al., 2009; Pan et al., 2011).

Genetic components have been shown to be a prime predisposing factor for T2DM. The IGF2BP2 gene is located on chromosome $3 q 27.2$ within a region linked to type 1 diabetes (T1D), type 2 diabetes (T2D), and diabetic nephropathy (DN) (Gu et al., 2012). IGF2BP2 has been found to be associated with decreased insulin secretion, which plays a role in T2DM. rs4402960 and rs1470579 of IGF2BP2 gene associated with T2DM susceptibility by comprehensive genetic association research (Rao et al., 2016). The gene encoding for transcription factor 7-like 2 (TCF7L2) is the strongest T2DM candidate gene discovered to date (Chen et al., 2018). The TCF7L2 gene is located on chromosome $10 q 25$ in a region of replicated linkage to type 2 diabetes (Damcott et al., 2006). The TCF7L2 variant rs7903146 appears to affect the risk of T2DM, at least in part, by modifying the effect of incretins on insulin secretion (Villareal et al., 2010). Molecular defects of hepatocyte nuclear factor 1B (HNF1B) are associated with a multiorgan disease, including diabetes (maturity-onset diabetes of the young 5) and kidney abnormalities (Dubois-Laforgue et al., 2017). In this study, we found the 3'UTR SNP (rs2229295) in the HNF1B gene was associated with the susceptibility of T2DM (Goda et al., 2015).

Hence, in this study, two bioinformatics tools, miRanda and TargetScan were used to determine the interaction between miRNAs present in chicken and IGF2BP2, HNF1B, and TCF7L2 based on miRanda score and context score++ percentile, respectively. The results predicted a strong association between them (Table 1). This interaction study indicated that the consumption of meat, especially chicken, would cause epigenetic modification of the genes involved in diabetes through the miRNAs present in chicken meat. Accordingly, prediabetics and those who have diabetes should avoid consuming chicken.

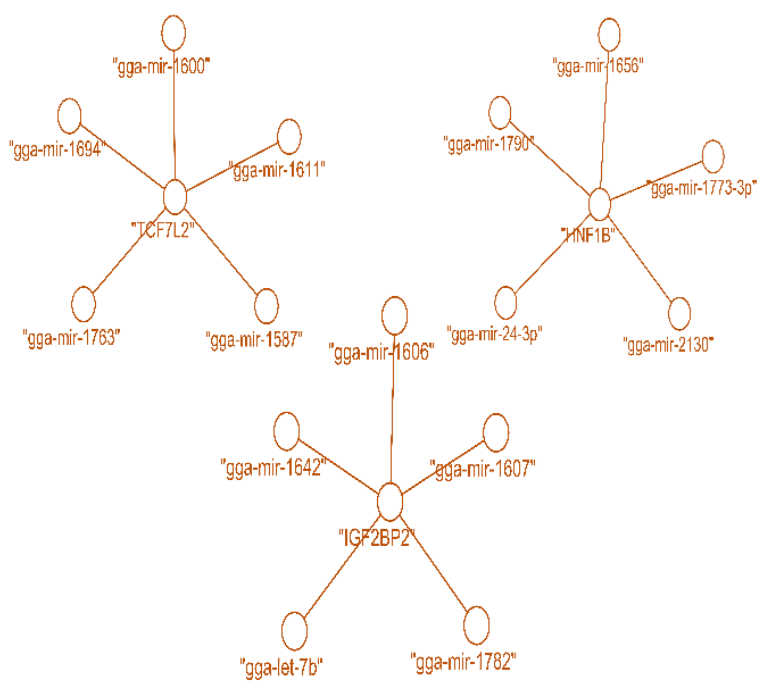

Figure: miRNA networking of chicken miRNAs and genes involved in diabetes. Network plotted and visualized utilizing miRNet. 
Table 1: mRNA-miRNAs interaction table with corresponding interaction score

\begin{tabular}{|c|c|c|c|}
\hline Gene & miRNAs & miRanda score & TargetScan score \\
\hline \multirow[t]{5}{*}{ IGF2BP2 } & gga-mir-1606 & 174 & 70 \\
\hline & gga-mir-1607 & 165 & 66 \\
\hline & gga-mir-1642 & 169 & 64 \\
\hline & gga-mir-1782 & 177 & 35 \\
\hline & gga-let-7b & 178 & 95 \\
\hline \multirow[t]{5}{*}{ HNF1B } & gga-mir-24-3p & 181 & 95 \\
\hline & gga-mir-1656 & 174 & 94 \\
\hline & gga-mir-1773-3p & 167 & 85 \\
\hline & gga-mir-1790 & 165 & 92 \\
\hline & gga-mir-2130 & 167 & 68 \\
\hline \multirow[t]{5}{*}{ TCF7L2 } & gga-mir-1600 & 155 & 94 \\
\hline & gga-mir-1611 & 181 & 80 \\
\hline & gga-mir-1694 & 173 & 74 \\
\hline & gga-mir-1763 & 169 & 48 \\
\hline & gga-mir-1587 & 168 & 44 \\
\hline
\end{tabular}

gga: Gallus gallus (chicken)

\section{Conclusion}

Several miRNAs are present in chicken, and other meat may make a consumer susceptible to diabetes, as these miRNAs may epigenetically modify numerous genes associated with diabetes. Future research on cells, animals, and humans are needed to confirm further this finding that will be having clinical implication

\section{Limitation of study}

The current work is a preliminary study conducted employing the online available in silicon algorithms and databases. Thereby, the findings of this study are not confirmatory; hence, further in-vitro, in-vivo, and clinical studies are needed to confirm and validate the above findings.

\section{Acknowledgement}

The authors are grateful to the Northern Railway Hospital, Associated Sri Mata Vaishno-Devi Narayana Superspeciality Hospital (SVDNSH), 
Katra, J\&K, for providing funding, resources, and support for conducting the research

\section{Conflict of interest}

Declared none by the authors

\section{References}

Aune, D., Ursin, G., and Veierod, M.B. (2009). Meat consumption and the risk of type 2 diabetes: a systematic review and meta-analysis of cohort studies. Diabetologia 52: 2277-2287.

Chen, X., Ayala, I., Shannon, C., Fourcaudot, M., Acharya, N.K., Jenkinson, C.P., et al. (2018). The diabetes gene and Wnt pathway effector TCF7L2 regulates adipocyte development and function. Diabetes 67: 554-568.

Cho, S.B., Kim, S.C., and Chung, M.G. (2019). Identification of novel population clusters with different susceptibilities to type 2 diabetes and their impact on the prediction of diabetes. Sci. Rep. 9: 3329.

Damcott, C.M., Pollin, T.I., Reinhart, L.J., Ott, S.H., Shen, H., Silver, K.D., et al. (2006). Polymorphisms in the transcription factor 7-like 2 (TCF7L2) gene are associated with type 2 diabetes in the Amish: replication and evidence for a role in both insulin secretion and insulin resistance. Diabetes 55: $2654-$ 2659.

Dubois-Laforgue, D., Cornu, E., Saint-Martin, C., Coste, J., Bellanné-Chantelot, C., and Timsit, J. (2017). Diabetes, associated clinical spectrum, long-term prognosis, and genotype/phenotype correlations in 201 adult patients with hepatocyte nuclear factor 1B (HNF1B) molecular defects. Diabetes Care 40: 1436-1443.

Goda, N., Murase, H., Kasezawa, N., Goda, T., and Yamakawa-Kobayashi, K. (2015). Polymorphism in microRNA-binding site in HNF1B influences the susceptibility of type 2 diabetes mellitus: a population based case-control study. BMC Med. Genet. 16: 75.

Gu, T., Horová, E., Möllsten, A., Seman, N.A., Falhammar, H., Prázný, M., et al. (2012). IGF2BP2 and IGF2 genetic effects in diabetes and diabetic nephropathy. J. Diabetes Complications 26: 393398.

Guariguata, L., Whiting, D.R., Hambleton, I., Beagley, J., Linnenkamp, U., and Shaw, J.E. (2014). Global estimates of diabetes prevalence for 2013 and projections for 2035. Diabetes Res. Clin. Pract. 103: 137-149.

Hashimoto, N., and Tanaka, T. (2017). Role of miRNAs in the pathogenesis and susceptibility of diabetes mellitus. J. Hum. Genet. 62: 141-150.

Kobberling, J. (1982). Empirical risk figures for first degree relatives of non-insulin dependent diabetes. Genet. Diabetes Mellit. 201:.

Ortega, Á., Berná, G., Rojas, A., Martín, F., and Soria, B. (2017). Gene-Diet Interactions in Type 2 Diabetes: The Chicken and Egg Debate. Int. J. Mol. Sci. 18:.

Pan, A., Sun, Q., Bernstein, A.M., Schulze, M.B., Manson, J.E., Willett, W.C., et al. (2011). Red meat consumption and risk of type 2 diabetes: 3 cohorts of US adults and an updated meta-analysis. Am. J. Clin. Nutr. 94: 1088-1096. 
Poulsen, P., Grunnet, L.G., Pilgaard, K., Storgaard, H., Alibegovic, A., Sonne, M.P., et al. (2009). Increased risk of type 2 diabetes in elderly twins. Diabetes 58: 1350-1355.

Poulsen, P., Kyvik, K.O., Vaag, A., and Beck-Nielsen, H. (1999). Heritability of type II (non-insulindependent) diabetes mellitus and abnormal glucose tolerance--a population-based twin study. Diabetologia 42: 139-145.

Pradhan, P., Upadhyay, N., Tiwari, A., and Singh, L.P. (2016). Genetic and epigenetic modifications in the pathogenesis of diabetic retinopathy: a molecular link to regulate gene expression. New Front. Ophthalmol. 2: 192.

Rana, S., Kumar, S., Rathore, N., Padwad, Y., and Bhushana, S. (2016). Nutrigenomics and its Impact on Life Style Associated Metabolic Diseases. Curr. Genomics 17: 261-278.

Rao, P., Wang, H., Fang, H., Gao, Q., Zhang, J., Song, M., et al. (2016). Association between IGF2BP2 polymorphisms and type 2 diabetes mellitus: a case-control study and meta-analysis. Int. J. Environ. Res. Public Health 13: 574.

Tiwari, J., Gupta, G., Jesus Andreoli Pinto, T. de, Sharma, R., Pabreja, K., Matta, Y., et al. (2018). Role of microRNAs (miRNAs) in the pathophysiology of diabetes mellitus. Panminerva Med. 60: 25-28.

Vang, A., Singh, P.N., Lee, J.W., Haddad, E.H., and Brinegar, C.H. (2008). Meats, processed meats, obesity, weight gain and occurrence of diabetes among adults: findings from Adventist Health Studies. Ann. Nutr. Metab. 52: 96-104.
Villareal, D.T., Robertson, H., Bell, G.I., Patterson, B.W., Tran, H., Wice, B., et al. (2010). TCF7L2 variant rs7903146 affects the risk of type 2 diabetes by modulating incretin action. Diabetes 59: 479-485. 